Biblioteca Central "Vicerrector Ricardo A. Podestá"

Repositorio Institucional

\title{
Gerencia estratégica de costos
}

\author{
Año \\ 2015 \\ Autor \\ Licera, Gloria Silvina
}

Este documento está disponible para su consulta y descarga en el portal on line de la Biblioteca Central "Vicerrector Ricardo Alberto Podestá", en el Repositorio Institucional de la Universidad Nacional de Villa María.

CITA SUGERIDA

Licera, G.S. (2015). Gerencia estratégica de costos. Villa María: Universidad Nacional de Villa María 


\title{
IV CONGRESO DE ADMINISTRACIÓN DEL CENTRO DE LA REPÚBLICA
}

\author{
“INSPIRACIÓN E IDENTIDAD PARA LA INTEGRACIÓN DE LAS \\ ORGANIZACIONES"
}

VILLA MARIA - 12 Y 13 DE NOVIEMBRE DE 2015

GERENCIA ESTRATEGICA DE COSTOS

LICERA GLORIA SILVINA. AUTORA Y EXPOSITORA 


\section{GERENCIA ESTRATEGICA DE COSTOS}

PALABRAS CLAVE: Gestión - Estrategia - Costo - Valor - Control

\section{Las nuevas necesidades de Información}

Es evidente, y así se pronuncia una gran cantidad de expertos, que un sistema de información de control y comprobación es insuficiente para las empresas que operan en la nueva economía, con un objetivo claro de creación de valor.

La nueva economía está sustentada, en el conocimiento, en la información y en las expectativas sobre ese conocimiento. Hasta ahora, el sistema de información contable, eminentemente de carácter económico-financiero, recoge información valorada en términos históricos, con escasa participación de las expectativas, lo que plantea dos interrogantes:

¿Ofrece el Sistema de Información Contable toda la información necesaria para cumplir con su fin?

¿Debe recoger el Sistema de Información Contable información sobre expectativas?

Lo que lleva a una tercer cuestión: ¿Es útil la información contable en este contexto?. Es evidente que las respuestas a tales preguntas no son sencillas y pueden tener mucho de compromiso político e intereses encontrados. No obstante se tratará de dar una respuesta a las mismas.

En primer lugar, hay que tomar en consideración que dado el impacto social que la contabilidad tiene, por el conjunto de agentes que, como ya se ha mencionado, se ven afectados (propietarios, trabajadores, clientes, proveedores, administraciones, etc.), eran necesarias un conjunto de reglas que permitieran cierta homogeneidad y comprensión de la misma, lo que llevó ya desde hace tiempo a una regulación específica. Esto es lo que ha ocurrido prácticamente en todo el mundo.

No cabe duda que la normativa contable es insuficiente en la nueva economía. Son insuficientes por diferentes motivos entre los que cabe citar, sobre todo, el hecho de 
tratarse de un sistema de información de carácter histórico que, por tanto, no recoge expectativas. Como señalan Cañibano y García-Ayuso (2000), "la creación de valor está cada vez menos relacionada con la acumulación y gestión de activos materiales y más con la generación y mantenimiento de ventajas competitivas relacionadas con los activos intangibles. En la medida en que las normas de contabilidad no permiten el reflejo de estos activos en balance, la estimación contable del valor de la empresa .. y de su capacidad de generación de riqueza ... aparecen sesgados a la baja.."

De lo que acabamos de decir no se puede desprender, en modo alguno, que la información contable no sea útil. Significa tan sólo que la vertiente "hacia el mercado", la que tiene que ver con las expectativas $y$, por ende, la que hace referencia al capital intelectual y la estrategia, no aparece en dicha información. La función de la contabilidad sigue siendo, sobre todo, de control y organización, debido a las bases sobre las que se asienta, sólo muy parcialmente de gestión y análisis y, escasamente de estrategia.

Para lograr una modificación sobre lo expresado, requiere satisfacer, a lo menos, cuatro condiciones fundamentales:

a) Una comprensión a la vez sintética y analítica, es decir, una comprensión del estado global del "sistema-empresa" a la vez que un conocimiento permanente de la influencia que cada una de las variables claves de los procesos que constituyen el sistema, tiene en ese estado global.

b) Un conocimiento permanente actualizado del estado del entorno y de sus probables tendencias de cambio; para poder mantener una adecuada relación empresa - entorno que permita ese ajuste vital continuo y evite sorpresas que pudieran resultar extremadamente peligrosas.

c) Un conocimiento claro, realista y práctico de las capacidades que posee la empresa y sus posibilidades de acrecentarlas, tanto en relación a las capacidades de sus miembros (equipos de dirección, como equipos de operación) como a la de sus recursos técnicos y de capital.

d) Una capacidad de generación de respuestas rápidas a los problemas de viabilidad y adaptación. Esto implica las tres condiciones anteriores y capacidad de creatividad e innovación. 
Siguiendo el razonamiento anterior, y partiendo de la concepción del modelo contable como una abstracción de la realidad, se ha entendido que existen distintos niveles de abstracción, y se propone que debería existir una clara relación entre los distintos niveles de abstracción a fin de coordinar esfuerzos. El nivel cotidiano es el primero, hace a la práctica de la contabilidad: oficina, manuales, etc. El segundo nivel es el técnico y tiene que ver con actividades desarrolladas con los registro de datos (proceso contable). El tercer nivel es el tecnológico (que tiene que ver con el análisis e interpretación de la contabilidad, o sea el desarrollo de las aplicaciones contables. El cuarto nivel está íntimamente relacionado con lo científico.

Es en el cuarto nivel donde se entiende que está el punto de partida para la redefinición de la contabilidad desde el conocimiento contable, partiendo de las teorías, el objeto de estudio, el lenguaje y el desarrollo del pensamiento contable, bajo el modelo de estrategia empresarial.

La contabilidad debe plantear un sistema de información basado en principios que generen mayor utilidad, ya que esta es una forma para que los entes creen valor. Como indica Kaplan y Norton (1997) "las estrategias, que son las únicas formas sostenibles que tienen las organizaciones de crear valor, están cambiando, pero las herramientas para medirlas no".

He aquí el motivo por el cual no debemos quedarnos con la simple técnica del ejercicio contable, sino que debemos estar ávidos de conocimiento, generando modelos en los que convivan los mecanismos del proceso contable con la lógica de los procesos estratégicos.

Lo allí discutido me sirve para reflexionar sobre el papel de la contabilidad en lo que ya se viene denominando nueva economía o economía del conocimiento, una economía fuertemente basada en la tecnología y la información y de marcado carácter globalizador. En una economía de estas características el papel de las empresas o, como otros prefieren denominar, negocios, se ve fuertemente mediatizado por la cantidad y calidad de la información, así como por la agilidad con que ésta llega a la 
misma para la toma de decisiones, al margen de otras consideraciones también de alto interés que llevan a cambios de hábitos y de comportamiento.

¿Cumple la contabilidad, en este nuevo entorno, con el rol de vehículo de información económica que de ella se espera?. Esta pregunta, que se encuentra últimamente latente en todos los foros en que participan los contables, requiere de una reflexión sobre el papel que hemos de asignar a la misma

Es suficientemente conocido que la contabilidad es uno de los sistemas de información posibles en la empresa y, por tanto, un instrumento a disposición de los interesados en la misma. Ciertamente es el sistema de información económico-financiera por excelencia. Por qué ello es así sería laborioso de tratar aquí y poco aportaría al objetivo planteado, baste argumentar que se trata de un sistema muy estructurado, que emplea un lenguaje que, una vez conocido, tiene ventajas indiscutibles para la explicación de los fenómenos y actividades empresariales, y con una relación costo-beneficio asumible, aspectos estos, junto a otros, avalados por la perseverancia de este sistema de Información a lo largo del tiempo.

Sin embargo, el devenir de esta disciplina científica, particularmente en los últimos 60 años, y sobre todo a partir de lo que se ha denominado teoría positiva de la contabilidad, ha generado cierta ansia sobre el poder explicativo de la misma con relación al comportamiento de los usuarios de la información empresarial. Esta tendencia, muy arraigada en el mundo anglosajón, ha pretendido que el sistema informativo contable fuera capaz de explicar o justificar por qué los usuarios de la información contable toman un tipo de decisiones y no otras con respecto a la empresa o negocio, por ejemplo, la relación existente entre la información contable publicada por la empresa y su cotización en el mercado de valores y, por tanto, la decisión de invertir o no en la misma.

Sin embargo, esta tendencia "hacia el mercado" olvida aquella otra para la que se concibió la contabilidad, la del control. Esto es, la contabilidad es un sistema de información que, tanto en el ámbito interno, como externo, tiene una función de comprobación, de gobierno, de dirección. Es importante no olvidar esto. El sistema contable actual, basado en los principios y criterios sobre los que hoy día se asienta, 
fundamentalmente el de costo histórico y devengado, está concebido como un sistema de información para el control y gobierno de las empresas o negocios.

Otra cosa muy distinta es si este sistema de información es hoy suficiente, si requiere ser ampliado o si debiese crearse un sistema adicional, complementario al mismo. Indudablemente, en la sociedad del conocimiento y la tecnología no cabe cruzarse de brazos. Se trata de dar soluciones coherentes dentro de un contexto regulado en donde se han de tomar en consideración los dos aspectos que se pretenden conjugar, control y estrategia, pero a sabiendas de que las herramientas no tienen porque ser forzosamente las mismas.

Al sistema contable actual no se le debe pedir más de lo que se le pide. Se trata de un sistema de regulación y control soportado en bases que, caso de cambiarse, difícilmente podrían cumplir su fin. ¿Significa eso que no se puede hacer nada para recoger las estrategias? En absoluto. Se debe estructurar un nuevo sistema de información para la valoración y gestión de la empresa que complete y complemente el anterior y se sirva de sus reglas y criterios para dar contenido y coherencia al mismo.

\section{Alcance de la Contabilidad Estratégica}

Nos encontramos ante lo que se denomina la era del conocimiento y en donde el cambio se vislumbra como permanente y duradero en términos de tiempos y resultados que deseamos obtener.

Nuestro sistema contable tradicional tiene como principal objetivo el estar orientado al tercero usuario de la información contable, llámese, bancos, estado, inversores, etc. los cuales se pueden nutrir, a partir de la información contable que surge de un balance, de una serie de datos para la toma de decisiones. Pero esta información adolece de un gran inconveniente, es relativa al pasado, lo cual en cierto sentido la inhabilita para tomar decisiones relativas al futuro.

Así el principal objetivo de la contabilidad es brindar información oportuna, veraz, confiable, pero orientada a la toma de decisiones.

Es decir nos encontramos ante una disyuntiva, existen sistemas contables que registran el pasado y con los cuales, debemos o intentamos nutrirnos para la toma de decisiones 
futuras que, en el mundo actual de permanente cambio es, a criterio de los especialistas, insuficiente para cumplir con esos objetivos, criterio al se adhiere.

Las decisiones son acciones que se tomarán en el futuro, ya sea cercano o lejano, las cuales deben estar sustentadas en información. Para ello debemos realizar la distinción entre, dato información y conocimiento. El dato es hecho aislado que describen la realidad y/o circunstancias tempo espaciales. La información es la sistematización de los datos en forma lógica y ordenada. El conocimiento es el trabajo que se realiza sobre esa información obteniéndose así un desarrollo posterior que lo valida como tal.

Puestas las cosas así, la toma de decisiones tanto a nivel gerencial como operacional requiere de información obtenida de sistemas que permitan formular pautas y criterios los que reunidos y sistematizados nos posibiliten orientar las acciones hacia el futuro.

A partir de esta idea macro se han desarrollado una serie de técnicas y enfoques que vienen a solucionar, en parte, la disyuntiva planteada y nos orientan a la obtención de información que se convierte en vital para la toma de decisiones de los usuarios que controlan los recursos.

La contabilidad estratégica se puede definir como "el sistema de información que incluye información financiera y no financiera para la toma de decisiones que soporta, mantiene e incrementa la posición competitiva de una organización" .la contabilidad estrategia, en la toma de decisiones, reemplaza a la tradicional, que puede mostrar mucho pero oculta lo más importante, porque las empresas no solo son números sino también hay que tomar en cuenta que hay muchos otros factores que la afectan.

El futuro no se prevé. El futuro se construye, se diseña. Ser competitivo sostenible es anticiparse para el futuro.

\section{GERENCIA ESTRATEGICA DE COSTOS}

La Gerencia Estrategia de Costos busca un nuevo enfoque respecto de la contabilidad tradicional, formulando una visión estratégica cuyo objetivo es alcanzar un grado de integración entre los costos y la cadena de valor. 
Partiendo del convencimiento de que la contabilidad no es un objetivo en sí mismo, sino que es únicamente un medio para ayudar a alcanzar el éxito empresarial y a partir de este concepto inferir que los sistemas de costos deben ser evaluados en función del papel que se espera que cumplan, esto implica que se deben desarrollar instrumentos de control gerencial sistemáticamente elaborados.

Al realizar una comparación ente la contabilidad tradicional y la gerencia estratégica de costos, podemos observar que ambas tienen objetivos y características distintas.

La modalidad de análisis de la contabilidad tradicional es en función de los productos y funciones, con marcado enfoque hacia lo interno. La gerencia estratégica de costos los analiza en función a las diferentes etapas de la totalidad de la cadena de valor.

En cuanto al comportamiento de los costos, la contabilidad tradicional es básicamente una función del volumen de producción, distinguiendo costo variable, costo fijo, costo combinado, etc., en tanto en la gerencia estratégica de costos se lo considera una función de las selecciones estratégicas, tanto las estructurales como las funcionales.

Como se puede definir la gerencia estratégica de costos? La Gerencia Estratégica de Costos es un proceso cíclico, continuo de formulación de estrategias, comunicación de estas estrategias, desarrollo y utilización de tácticas para implementarlas, y el desarrollo y establecimiento de controles para supervisar el éxito de las etapas anteriores.

Los componentes básicos de la gerencia estratégica de costos son tres:

1. Análisis de la cadena de valor

2. Análisis del posicionamiento estratégico

3. Análisis de las causales de costos.

\section{Cadena de Valor}

Michael Porter, desarrolló el concepto, en su obra Estrategia Competitiva (1980), el cual se basa en que cada unidad de negocio debe desarrollar una ventaja competitiva continua, basándose en el costo, en la diferenciación o en ambas cosas.

El análisis de la cadena de valor comienza con el reconocimiento de que cada empresa o unidad de negocios, es "una serie de actividades que se llevan a cabo para diseñar, 
producir, comercializar, entregar y apoyar su producto". Al analizar cada actividad de valor separadamente, los administradores pueden juzgar el valor que tiene cada actividad, con el fin de hallar una ventaja competitiva sostenible para la empresa.

Al identificar y analizar las actividades de valor de la empresa, los administradores operan con los elementos esenciales de su ventaja competitiva, ya que la eficiencia y eficacia de cada una de las actividades afecta el éxito de la empresa en su estrategia.

Las actividades las podemos dividir en dos tipos, las principales y las de apoyo. Las primeras, son la logística interna, operaciones, logística externa, marketing, y el servicio. Esta serie de actividades las podemos imaginar como una corriente de actividades relacionadas, empezando desde la llegada y el almacenamiento de las materias primas o insumos para los procesos de producción, su transformación en productos finales que se expiden, las actividades de comercialización y venta para identificar, alcanza y motivar a los clientes o grupos de clientes y las actividades de servicio para prestar apoyo al cliente y/o al producto después de la compra.

Luego las actividades de apoyo, como su nombre lo indica prestan un respaldo general y especializado a las actividades primarias

Estas son la administración, de compras, de recursos humanos, el desarrollo tecnológico, y la infraestructura. Debemos considerar a estas como funciones empresariales ya qué sin ellas no existiría una organización y juntamente con el grado de vinculación con las principales, conforman lo que se denomina el análisis de la cadena de valor, que como herramienta en la formulación de estrategias, exige que los administradores no solo analicen por separado cada actividad de valor con todo detalle, sino que también examine las vinculaciones criticas entre las actividades internas.

Al referirnos al tema costos este enfoque es diferente al desarrollado por la contabilidad tradicional, la cual se basa en el concepto de valor agregado, el que supone maximizar la diferencia entre las compras y las ventas. Es decir que el valor agregado fija su atención en las funciones internas de la Empresa, comienza con las compras a los proveedores y termina con los costos pagados por los clientes (ventas).

La gerencia estratégica de costos, nos explica que al fijar un enfoque tan estrecho, como lo es el valor agregado, la Empresa, pierde una serie de ventajas y oportunidades que comienzan antes de la compra y terminan más allá de la venta al cliente. 


\section{Metodología de la cadena de valor}

Para construir una cadena de valor los pasos fundamentales son:

1- Identificar la cadena de valores de la industria y asignarles costos, ingresos y activos a las actividades de valor.

2- Diagnosticar cuáles son las causales de costos que regulan cada actividad de valor.

3- Desarrollar una ventaja competitiva sostenible.

La ventaja competitiva no puede ser analizada dentro de una empresa como un todo, sino que debe descomponerse la cadena de valores en sus diferentes actividades estratégicas, ya que cada una incurre en costos, genera ingresos y está ligada a activos, separándose en aquellas que representan un importante porcentaje de los costos operativos, o si el comportamiento de costos de las actividades son diferentes, o si los competidores las ejecutan en forma diferente o si tiene alto potencial para crear diferenciación. A partir de este desarrollo se puede calcular el rendimiento sobre activos para cada actividad de valor.

A partir del análisis anterior debemos identificar las causales de costos que expliquen las variaciones de costos en cada actividad de valor. En la contabilidad gerencial tradicional el costo tiene una sola causal, la cual es el volumen de actividad o de producción. En la cadena de valores el concepto del volumen de producción, capta muy poco de la riqueza del comportamiento de los costos. Coexisten múltiples causales de costos las cuales además difieren a través de las actividades de valor. Estas pueden ser estructurales o ejecucionales. Las primeras podemos definirlas como aquellas que al ser elegidas por la empresa impulsan el costo del producto.

Estas son:

a. La escala, es decir la cuantía de la inversión que se va a realizar en áreas de fabricación, investigación, recursos de marketing, etc.

b. Extensión, es decir el grado de integración vertical. 
c. Experiencia, se refiere al número de veces que en el pasado la empresa ha realizado lo que ahora está haciendo.

d. Tecnología, nos referimos a los métodos tecnológicos utilizados en cada etapa de la cadena de valores.

e. Complejidad, se refiere a la amplitud de la línea de productos o servicios que se ofrecerán a los clientes.

En cuanto a las segundas, ejecucionales, son aquellas que son decisivas para establecer la posición de costos de una empresa y que dependen de sus habilidades para ejecutarlas con éxito. Dentro de la lista de estas causales podemos mencionar las siguientes:

a. Compromiso con el grupo de trabajo.

b. Gerencia de calidad total.

c. Utilización de la capacidad.

d. Eficiencia en la distribución de la planta.

e. Configuración del producto.

f. Aprovechamiento de los lazos existentes con proveedores y/o clientes a través de la cadena de valores de la empresa.

El tercer paso, luego de identificar la cadena de valor y de diagnosticar las causales de costos de cada actividad, se puede ganar una ventaja competitiva sostenible por medio de dos formas:

I- $\quad$ Controlando las causales de costos mejor que los competidores, es decir se puede analizar las causales y mejorar el rendimiento de las causales de costos, se puede emplear el Benmarching, sobre los competidores, sabiendo que esta opción debe ser siempre revisada y reconsiderada en el corto plazo, ya que el competidor operará de la misma forma.

II- Reconfigurando la cadena de valores: lo que implica que mientras se recompone las causales de costos habrá que redefinir la cadena de costos en las actividades en donde sea más necesario. 


\section{Posicionamiento estratégico}

Se analizará en este punto cual es el papel de la información contable dentro del negocio, el que en principio es el de facilitar el desarrollo y la implantación de estrategias.

Esto es lo que distingue a la gerencia estratégica de costos respecto de la contabilidad gerencial tradicional. La relación existente entre costos y las estrategias, explicadas anteriormente, se resuelve por la influencia que tienen en cada tipo de estrategia elegida, la generación de costos y por consiguiente el control que se debe efectuar en el proceso gerencial.

Las estrategias difieren en los diversos tipos de organización y los controles debieran adaptarse a los requerimientos de las estrategias elegidas.

La ligazón entre los controles y las estrategias tienen su origen en las siguientes ideas: Para la ejecución eficaz, diferentes estrategias requieren diferentes prioridades de tareas, factores claves para el éxito, experiencias y perspectiva y comportamientos.

Los sistemas de control son unidades de medidas que influyen el comportamiento de las personas cuyas actividades están siendo medidas.

Por ello debiera realizarse un diseño de sistemas de control acorde a la coherencia entre la estrategia y la influencia sobre las personas.

El análisis estratégico se basa en los conceptos mencionados, relativos a la Visión, Misión, Objetivos, y Estrategias que operan a modo de vértice principal en la elaboración de controles y de la posibilidad de obtener ventajas competitivas sostenibles y cuidado en la cadena de valor.

Uniendo conceptos deberíamos insistir en que el control gerencial depende de la estrategia elegida para obtener la ventaja competitiva y de las opciones que se escojan respecto de la cadena de valor. Esto es a la luz de la misión formulada y de la estrategia elegida.

Es decir una empresa cuya misión está definida dentro del marco de un mercado maduro, con productos no diferenciados y con una estrategia de liderar en costos, los costos del diseño del producto deberán ser una herramienta de importancia fundamental. 
Así cada organización y dependiendo de la misión formulada y de la estrategia elegida deberá asignarle un sistema de control tomando en consideración también otros factores como son, la tecnología, la cultura, el contexto externo del ambiente, etc.

\section{Causales del costo}

El tercer elemento constitutivo de la gerencia estratégica de costos, es el concepto de las causales del costo.

Estas toman un lugar preponderante en la gerencia estratégica de costos, debido al enfoque estratégico que se le da a estas causales y por sobre todo por la unión, en la que se relacionan la cadena de valor y el posicionamiento con este tercer elemento.

Es así que en la gerencia estratégica de costos se acepta el hecho de que los costos son impulsados por múltiples factores, esto también explica las distintas variaciones de costos en cada actividad.

Como mencionamos anteriormente en la contabilidad gerencial, el costo es una función exclusiva del volumen de producción. De ahí se desprenden una serie de análisis relativos a este factor, dentro del cual mencionaremos a modo de ejemplo los siguientes, la división entre costo fijo y variable, la relación volumen, costo, utilidad, el control presupuestario, etc.

Es aquí donde la gerencia estratégica de costos genera la novedad de no solo considerar al volumen de producción como causal del costo sino que además incorpora una serie de modelos más avanzados en la relación de la causalidad del costo, como lo vimos anteriormente.

A modo de mención diremos que las causales son divididas en dos: causales estructurales y de ejecución.

No se abundará en ellas, solo afirmar la consideración que la gerencia estratégica de costos avanza sobre la contabilidad gerencial al enfocar los temas desde otro ángulo y fundamentalmente posiciona al costo dentro del espectro de toma de decisiones en la empresa de manera integral y abre un camino distinto hacia herramientas de gestión, que pueden ser utilizadas en pos de una mejora en la administración de una organización. 


\section{APLICACIÓN DE LA GESTIÓN ESTRATÉGICA DE COSTOS}

La Gerencia Estratégica de Costos tiene como fortaleza la de alcanzar los objetivos planteados desde un enfoque estratégico y consolidar un sistema distinto y fundamentalmente útil en un momento en donde la realidad es constantemente modificada.

Ya se ha definido la gerencia estratégica de costos como un proceso cíclico, continuo de formulación de estrategias, comunicación de estas estrategias, desarrollo y utilización de tácticas para implementarlas, y el desarrollo y establecimiento de controles para supervisar el éxito de las etapas anteriores. La información contable es necesaria para cada etapa de este ciclo.

En la primera la información contable es la base para realizar el análisis financiero, el cual constituye un elemento del proceso de evaluación de estrategias alternativas. En la segunda, los informes constituyen una de las formas importantes por las cuales estas estrategias se comunican a la organización en su conjunto. En la tercera, en el desarrollo de las tácticas específicas que apoyen la estrategia en su conjunto y de su implementación, los informes financieros confeccionados sobre la base de la información contable, son uno de los elementos que sirve de sustento a las tácticas, para lograr que se cumplan con los objetivos deseados. En la cuarta, la evaluación del desempeño de los gerentes o de las distintas unidades de negocio habitualmente depende de la información contable.

Desde esta perspectiva, en primera instancia surge el costo como parámetro fundamental para la determinación del valor de agregado, para en una instancia posterior y a través de la gestión estratégica de los mismos, contribuir al crecimiento de la organización en el largo plazo, por ende a la determinación y mantenimiento del valor de la organización en el tiempo.

En la visión clásica, el concepto de valor asumía su máxima expresión en el denominado valor agregado, cuyo componente principal era el factor trabajo (horas hombre y máquina), para pasar a una visión diferencial dada por uno de los objetivos de las organizaciones cual es maximizar la diferencia entre compras y ventas, en donde el costo del producto aparece como factor clave para la determinación del precio de venta. 
Ahora bien, el avance de las Ciencias de la Administración, de la mano con la Contabilidad, le otorgan al valor agregado una connotación mucho más limitada.

Todo ello lleva a cambiar la visión del Costo (Sader G, 2011), ya que no sólo son importantes para su análisis el volumen de producción, su composición y su comportamiento (fijo o variable), sino que el costo pasa a formar parte de la estrategia de la organización que conduce a su posicionamiento estratégico teniendo en cuenta además las distintas etapas de la cadena de valor de cada organización. Entonces, el valor de una organización ya no puede medirse en función al valor agregado al costo de los recursos, sino que debe ponderarse en función a la manera en que el capital intelectual es gestionado sobre esos recursos. $Y$ en todo este proceso, es el decisor quien debe generar ese posicionamiento estratégico, utilizando las herramientas que le brinda la contabilidad de gestión y específicamente la Gerencia Estratégica de Costos, a fin de optimizar el resultado de la acción conjunta de la información (sinergia) con su inteligencia interpersonal (empatía), en un análisis temporal donde posición, retrodicción y proyección están en permanente interacción.

\section{El rol del sistema de información contable y su relación con la GEC y los ciclos gerenciales desde dos perspectivas:}

\section{Perspectiva sinérgica}

Se entiende por sinergia al resultado de la acción conjunta de dos o más causas, cuyo efecto conjunto es mayor al resultante de la simple suma de estas. En ese sentido, los sistemas de información con respecto al comportamiento de las personas que lo usan no son neutros. $Y$ puesto que los individuos no reaccionan pasivamente a la información, el diseño es un factor de influencia decisivo en la consecución de los objetivos. Según cuál sea su diseño y forma de utilización, puede estar sirviendo a unos intereses o a otros, la información puede llegar a ser manipulada y a convertirse en instrumento de legitimación (Carmona Moreno, 1993).

En la GEC, el diseño del sistema de información estratégica debe considerar el sano equilibrio entre los tres elementos conceptuales que influyen en los costos (la cadena 
de valor, el posicionamiento estratégico y sus generadores) y a esto se lo denomina perspectiva sinérgica.

Entonces, lo importante en el análisis y determinación de los costos desde una perspectiva estratégica es que la combinación de los tres elementos mencionados accionando conjuntamente en el costo, tenga un efecto en términos de eficiencia y eficacia superior del que pueda generarse de considerarlos en forma individual. No se puede efectuar un análisis separado de cada elemento conceptual sin evaluar la sinergia en el conjunto, que es lo que en definitiva contribuye al cumplimiento de la estrategia definida.

Perspectiva empática: la inteligencia del decisor, la definición y el control de la estrategia organizacional

Para mejorar la manera en que el sistema de información contable impone su influencia, es necesario también que el decisor asuma su propia visión con una capacidad particular: la empatía. En este sentido, la teoría de las inteligencias múltiples aporta un modelo propuesto por Howard Gardner (1983) en el que la inteligencia no es vista como algo unitario, que agrupa diferentes capacidades específicas con distinto nivel de generalidad, sino como un conjunto de inteligencias múltiples, distintas e independientes.

La empatía, llamada también inteligencia interpersonal en la teoría de las inteligencias múltiples, es la capacidad cognitiva de percibir en un contexto común lo que un individuo diferente puede sentir.

Gonzalo Angulo y Carmona Moreno (1995), afirman con respecto al sistema de información contable, que su misión no se limita a recoger información, sino a planificarla, elaborarla e incluso crearla. La imagen del Sistema de Información Contable adaptándose a las necesidades de los decisores y siendo neutral en las decisiones es sólo ficción. El Sistema de Información Contable a menudo impone su propia visión de la realidad y condiciona el proceso de decisión y control.

Igualmente concuerda con este planteamiento el doble acondicionamiento que para Kaplan (1984) ha de tener el sistema informativo contable: no solo demandará una determinada información, sino que influirá en la fijación de los objetivos de control, en el 
cumplimiento de tales objetivos a través de la motivación, la valoración de su actuación, y de las medidas correctoras que se tomen.

Las acciones mencionadas de fijar, motivar, cumplir y valorar requieren que el decisor posea capacidades y actitudes tendientes a resolver problemas, y para ello su ubicación en el plano del "otro" (quien es el objeto de la motivación, del control y de la valoración) mejorará la estrategia organizacional en relación al desarrollo de los cuatro ciclos ya mencionados:

1. Formulación de la estrategia

2. Comunicación de la estrategia

3. Desarrollo y utilización de tácticas para implementar las estrategias

4. Establecimiento de controles para supervisar las etapas de la estrategia y por ende alcanzar los objetivos estratégicos.

En Shank y Govindarajan (2005), se muestran casos específicos de empresas con problemas en los generadores de costos, posicionamiento en la cadena de valor y posicionamiento estratégico. Esta propuesta va mas allá de la casuística, intenta explicar qué principios, pilares, conceptos, secuencias y escenarios, deben estar en la mente de quien decide para eficientizar sus cursos de acción; todo ello poniendo énfasis en las actividades como base del valor organizacional y expandiendo su análisis al contexto en que la organización se encuentra inmersa.

La intención, en este análisis, es mostrar el avance de la Contabilidad Gerencial sustentado en pilares de la GEC. En este sentido, la base está dada en la definición de las actividades, lo que lleva a determinar sus generadores de costos.

Al respecto, la Contabilidad Gerencial, aporta eficientes sistemas de gestión de costos y de evaluación de performance con base en las actividades.

Siguiendo a Horngren, Datar y Foster (2007), Kaplan y Cooper (1999), Pérez Castillo y Rodríguez Hernández (2011), se pueden encontrar distintos sistemas, que combinados, pueden contribuir a los objetivos de la GEC, ellos son el Costo Basado en Actividades o Subactividades (ABC, Activity Based Cost), la Administración Basada en Actividades (ABM, Activity Based Management) y la Presupuestación Basada en Actividades (ABB, Activity based Budgeting). 
El $A B C$ surge como un sistema de costo dirigido a la estrategia organizacional. En este sistema, es de vital importancia definir las actividades como áreas funcionales que consumen recursos, para luego relacionar los productos con esas actividades (los productos consumen las actividades).

Con ello se logra diferenciar aquellas actividades que generan valor de las que no generan valor, dejando al decisor la oportunidad de tomar decisiones al respecto, retroalimentando su estrategia.

La implantación del ABC está facilitada por la previa existencia de un ambiente favorable, donde se tiene transparente la cadena de valores y ya esbozados el posicionamiento estratégico y direccionadores de costos.

El Just in time, implica eficiencia en el manejo de los inventarios a través de medidas que mantengan los materiales y productos el menor tiempo posible en almacenes, a fin de ahorrar costos (de administración- control y espacio- y de financiación), garantizando la entrega justo a tiempo en cada una de las etapas que va desde la materia prima al producto en manos del cliente $E \mathrm{E} A B M$, es más amplio que el $A B C$, ya que está más ligado a la gestión empresarial en su conjunto y no se limita sólo a la gestión de los costos, ya que complementa el $A B C$ con políticas de mejora continua hacia calidad total y sostenibilidad del el ciclo de vida de los productos. Su finalidad es aumentar la competitividad de la empresa. Se basa en análisis causa y efecto -a través de los generadores de costos-, actividades generan y no generan valor, calidad y satisfacción del cliente, copia de prácticas similares (benchmarking) o de las mejores prácticas (best practices) (Sader G. 2011).

El $A B B$ implica un avance de los principios del $A B C$ y del $A B M$ en materia de presupuesto de las actividades definidas. Implica un plan integral y coordinado, medido en términos financieros, de las operaciones y recursos de una organización para un determinado período de tiempo, elaborado en base a las actividades con la finalidad de lograr los objetivos fijados por la gerencia, propendiendo a la toma de decisiones en términos de eficacia, eficiencia y efectividad. 


\section{CONSIDERACIONES FINALES}

La evolución de la herramienta Contabilidad de Costos trajo aparejada la necesidad de desarrollar nuevos enfoques para el análisis del costo. La tradicional forma de acumular, asignar y clasificar los costos, si bien sigue siendo de utilidad, fue incorporando en el tiempo el análisis de la influencia de los procesos y de distintos actores, pasando así de una visión introspectiva a una visión estratégica donde todos los componentes de la cadena de valor de una organización deberán ser evaluados para lograr ventajas competitivas.

El decisor logrará desarrollar su estrategia en forma eficiente y eficaz a través tanto de su capacidad de analizar desde la perspectiva de otros actores todo el proceso estratégico (empatía), como de la habilidad de combinar la información sobre los tres elementos conceptuales del costo (cadena de valor, posicionamiento estratégico y sus generadores) de manera tal que de esa combinación se logren los mejores resultados.

\section{BIBLIOGRAFÍA}

CARMONA MORENO, S. La Crisis de los Sistemas de Contabilidad de Gestión: Una Perspectiva Española, en Carmona Moreno, S. (ed.): Cambio Tecnológico y Contabilidad de Gestión. Madrid, ICAC: 483-542. (1993)

GARRIDO BUI, S. (2003) Dirección Estratégica - Ed. Mc Graw Hill - España

GONZALO ANGULO, J.A.; CARMONA MORENO, S. (1995). Las Claves de Futuro en Contabilidad de Costes, en Contabilidad de la Empresa y Sistemas de Información para la Gestión. III Congreso Internacional de Costos y I de

KAPLAN, R. y COOPER, R. (1999). Coste y Efecto. Barcelona: Gestión 2000.

LAZZATTI, S. El Proceso Decisorio. Enfoque, Método y Participación. Ed. Macchi. Argentina. (1997)

LIMONE ARAVENA, A. Contabilidad y gestión del conocimiento en el contexto del nuevo enfoque en la teoría de la organización. Revista do mestradoi em administraçao e desenvolvimento empresarial. Impresión Universidade Estácio de Sá. (2003)

PEREZ CANTILLO, N., y Rodríguez Hernández, J. A. (2011). El costeo basado en actividades.

Recuperado

de http://www.elprisma.com/apuntes/administracion_de_empresas/costeobasadoenactivida des/ 
SADER, G., TISSERA P. La influencia de la Información contable estratégica en el cambio organizacional. Revista XXII Congreso Nacional del ADENAG. (2006)

SADER, G., TISSERA P. (2006). La influencia de la Información contable estratégica en el cambio organizacional. Revista XXII Congreso Nacional del ADENAG.

SHANK, J. y GOVINDARAJAN, V. (2005). Gerencia Estratégica de Costos.

VINITZKY, G. Estrategia y Sistemas de Información. Ed. Mc. Graw- Hill. Segunda Edición (1996) 